\title{
EFFETS DE TROIS TEMPÉRATURES NOCTURNES DE L'AIR SUR LES RENDE- MENTS DE LA TOMATE DE SERRE CULTIVÉE EN SOL ET EN NFT
}

\author{
CHRISTIAN CHOLETTE et DANIEL LORD
}

Département des Sciences Fondamentales, Université du Québec à Chicoutimi, Chicoutimi, Québec, Canada G7H 2B1. Reçu le 31 mai 1988, accepté

le 9 septembre 1988.

Cholette, C. ET LoRD, D. 1989. Effets de trois temperatures nocturnes de l'air sur les rendements de la tomate de serre cultivée en sol et en NFT. Can. J. Plant Sci. 69: $317-324$.

L'objectif de cet article est d'étudier l'effet d'un abaissement de la température nocturne de l'air d'une serre avec petits tunnels sur les rendements hâtifs et cumulatifs de la tomate (Lycopersicon esculentum Mill. 'Carmello') cultivée sur trois tiges en plein sol et en NFT. Les tomates ont été semées le 16 janvier 1984. Les plants ont été soumis à trois températures nocturnes de l'air $\left(17,12,7^{\circ} \mathrm{C}\right)$. Les traitements ont débuté le 24 février, soit au stade six feuilles déployées et première grappe en bouton. Ils se sont poursuivis pendant deux mois, jusqu'au moment où les conditions climatiques extérieures ne permettaient plus le maintien de la température nocturne de $7^{\circ} \mathrm{C}$. Les résultats démontrent, sous toutes les conditions, l'avantage de la culture NFT sur la culture en sol en terme de rendements totaux et vendables, alors que la fraction hâtive de ces mêmes rendements ne diffère pas significativement avec le type de culture. Le développement des plants est grandement réduit par de basses températures de nuit. Par exemple, la date d'ouverture de la moitić des fleurs de la première grappe est retardée de deux semaines à $7^{\circ} \mathrm{C}$. Le rendement hâtif est également significativement réduit par la diminution des températures de nuit, alors que le temps requis pour que les plantes se libèrent de la moitié de leur production totale est augmenté de deux semaines pour la culture en NFT et d'une semaine pour celle en plein sol. Les températures nocturnes de l'air contrôlent donc en bonne partie la vitesse de développement des plants de tomate et, conséquemment, la précocité du rendement en fruits vendables.

Mots clés: Tomate, serre, température de nuit, NFT, sol, rendement total et hâtif

[Night air temperature effects on yields of greenhouse tomato produced in soil and NFT. ]

Short title: Low night air temperature effects on tomato.

The objective of this research was to evaluate the effects of low night-air temperatures on the early and total yields of the tomato (Lycopersicon esculentum Mill. 'Carmello') grown with three stems in NFT and soil, in a greenhouse with small tunnels. Tomato was seeded on $16 \mathrm{Jan}$. and was grown under three night air temperatures $\left(17,12,7^{\circ} \mathrm{C}\right)$ for 2 mo after the sixth leaf had expanded and the first cluster was visitle, say from $24 \mathrm{Feb}$. to $15 \mathrm{Apr}$. Total and marketable yields were significantly higher in NFT than in soil, but there was no advantage for the early yield. The date at which half the flowers of the first cluster opened was 2 wk earlier for the $17^{\circ} \mathrm{C}$ treatment than for the $7^{\circ} \mathrm{C}$ treatment, indicating that low night-air temperatures reduce the speed of development. Low night-air temperature significantly reduced the early yield, while the time to half of total yield increased by a factor of $2 \mathrm{wk}$ in NFT and $1 \mathrm{wk}$ in soil. Night air temperatures show large effects on the speed of development of the tomato and the earliness of the marketable yield.

Key words: Tomato, greenhouse, night-air temperature, NFT, soil, early and total yields

Can. J. Plant Sci. 69: 317-324 (Jan. 1989) 
Il existe au Canada un grand nombre de petites communautés nordiques qui se retrouvent isolées des grands marchés horticoles. L'éloignement rend difficile l'approvisionnement de ces communautés en légumes frais, particulièrement durant les saisons froides de l'année. La production en serre représente une avenue intéressante pour combler ce besuin. Cependant, les coûts de chauffage accaparent une part élevée du budget de fonctionnement d'une production de légumes de serre cultivée en milieu nordique, ce qui n'est pas sans affecter la rentabilité de tels modes de production dans ces régions.

Pour des complexes de serre de faible dimension, il a été prouvé que la création d'un micro-climat adéquat pour la croissance des plantes en milieu nordique peut se faire à moindre coût par l'installation de petits ou de grands tunnels dans la serre (Gallagher et Vonarburg 1981). Ce système permet de diminuer significativement les pertes radiatives et conductives d'énergie, particulièrement la nuit. La présence des petits tunnels oblige toutefois le producteur à restreindre la hauteur de ses plants. La méthode de culture sur deux ou trois tiges est particulièrement bien adaptée à cet égard, puisqu'elle permet d'obtenir plusieurs grappes sur une hauteur de plant de deux à trois fois moindre qu'en culture sur une seule tige (Gallagher et al. 1981).

Toujours en vue d'abaisser les coûts de chauffage, il convient de réduire le plus possible l'écart thermique entre l'air de la serre et le milieu extérieur, particulièrement la nuit car cette période gruge la plus grande partie du budget de chauffage d'un producteur.

Les travaux précédents sur ce sujet montrent des résultats contradictoires pour la tomate de serre. Alors que certains rapportent que le maintien, pendant la nuit, d'une température du sol ou du substrat supérieure à celle de l'air minimise les effets négatifs des basses températures nocturnes sur la croissance et le rendement des tomates de serre (Verkerk 1966; Kaname et Itagi 1973; Jones et al. 1978; Morgan et O'Haire 1978; Ingratta 1980; Gosselin et Trudel 1983), d'autres affirment que ces effets négatifs demeurent significatifs malgré tout (Abdelhafeez et al. 1971; Moorby et Graves 1980; Orchard 1980; Papadopoulos et Tiessen 1983). Il en va de même pour la thermopériodicité, c'est-à-dire l'effet de la variation nycthémérale de la température du système radiculaire sur le développement des plantes. Certains auteurs soutiennent que cette périodicité est de prime importance (Hussey 1965; Papadopoulos et Tiessen 1983), alors que d'autres affirment le contraire (Lewis 1953; Calvert 1964). Il existe cependant un certain consensus autour de la nécessité de maintenir les racines à une température relativement élevée lorsque la température de l'air est abaissée.

Les travaux effectués jusqu'ici ont principalement porté sur des productions cultivées en plein sol selon la méthode de culture sur une seule tige. Quoique très répandue, la technique de culture en plein sol ne permet qu'un contrôle thermique approximatif de l'environnement du système radiculaire. De plus, pour des raisons de prévention des maladies avant tout et de productivité, nombre de producteurs se dirigent actuellement vers des techniques de culture hors-sol. Ceci devrait être d'autant plus vrai dans les régions nordiques, la disponibilité de sol adéquat pour une production horticole s'avérant limitée. L'objectif spécifique de cet article est d'étudier les effets, durant la période pépinière, d'un abaissement de la température nocturne de l'air d'une serre avec petits tunnels sur les rendements hâtifs et cumulatifs de plants de tomate cultivés sur trois tiges en plein sol et sur film nutritif (NFT) à solution recirculante (Cooper 1979).

\section{MATÉRIEL ET MÉTHODES}

Des graines de tomate (Lycopersicon esculentum Mill. 'Carmello') ont été semées le 16 janvier 1984 dans un mélange de tourbe-perlite-vermiculite (3:2:1). Les plants ont été fertilisés dès l'apparition des premières feuilles et repiqués trois semaines après le semis. Au 24 février, soit cinq semaines après les semis, les trois traitements de température nocturne de l'air choisis pour cette expérience $\left(17,12\right.$ et $\left.7^{\circ} \mathrm{C}\right)$ ont été appliqués suite à la répartition des plants dans chacune des platesbandes des deux systèmes de culture utilisés. La 
moitié des plants a été transplantée dans trois unités indépendantes de culture sur film nutritif (NFT) à solution recirculante (Cooper 1979) situées dans la moitié est d'une serre gothique de $8 \mathrm{~m}$ par 18 m. L'autre moitié des plants a été répartie dans les trois plates-bandes situées dans la moitié ouest. Ces derniers ont été rempotés la septième semaine, puis transplantés en plein sol la onzième semaine après le semis, soit six semaines après le début des traitements de température de nuit.

Le système NFT comprend trois plates-bandes doubles de $7.4 \mathrm{~m}$ de long, chacune alimentée indépendamment à partir de sa propre réserve de solution nutritive (Cooper 1979). La température de la solution est maintenue jour et nuit à $20 \pm 1^{\circ} \mathrm{C}$, alors que sa conductivité et son $\mathrm{pH}$, mesurés quotidiennement, sont respectivement maintenus à 0,02 $\mathrm{dS} \mathrm{cm}^{-1}$ et 6,5 . La solution circule dans chacune des gaines du système à un débit de $1,2 \mathrm{~L} \mathrm{~min}^{-1}$. La quantité de solution perdue par transpiration et évaporation est mesurée régulièrement et compensée par l'ajout de nouvelle solution ou par de l'eau pure, selon la conductivité. La solution est complètement renouvelée à toutes les trois semaines au minimum.

La culture en plein sol s'est aussi faite dans trois plates-bandes doubles de 7,4 $\mathrm{m}$ de longueur. La régie de fertilisation et d'irrigation suit les recommandätions du Conseil de productions végétales du Québec (1984) pour une culture de printemps de tomates de serre. Des gaines dans lesquelles circulaient de l'eau à $27^{\circ} \mathrm{C}$ ont été déposées sur le sol afin de le chauffer. La température était mesurée quotidiennement à $0.1 \mathrm{~m}$ sous sa surface du sol; elle est demeurée constante à $20 \pm 2^{\circ} \mathrm{C}$.

Certaines contraintes physiques nous ont obligé à réaliser un dispositif basé sur des blocs traitements plutôt que des blocs répétitions. Les répétitions, au nombre de quatre par traitement, sont distribuées dans les blocs traitements. Chaque plate-bande correspond donc à une unité expérimentale et contient 30 plants pour un total de 180 plants dans la serre. Toutefois, les mesures n'ont été prises que sur les 20 plants situés au centre de chacune des plates-bandes, cinq plants étant mesurés par répétition. Une analyse de variance à deux facteurs a été appliquée à ces données.

Tous les plants sont cultivés sur trois tiges. Cette technique de culture consiste à laisser pousser les premiers drageons qui se développent à l'aisselle de chacune des deux feuilles situées immédiatement sous la première grappe de fleurs. L'obtention d'un nombre de grappes donné, soit sept grappes sur la tige principale, cinq sur la seconde et trois sur la troisième dans le cadre de cette expérience, peut donc se faire sur un plant beaucoup plus petit que s'il n'avait qu'une seule tige pour porter les 15 grappes. Cette technique de culture doit obligatoirement être utilisée avec le système de culture sous doubles tunnels lorsque ces derniers sont de dimension restreinte (Gallagher et Vonarburg 1981).

Au coucher du soleil, chaque plate-bande est recouverte d'un film de polyester aluminisé de 15 $\mu \mathrm{m}$ d'épaisseur, cet écran thermique étant retiré au lever du soleil. Ce système possède l'avantage supplémentaire de permettre un contrôle précis de la variable "température nocturne de l'air" étudiée dans cette expérience. Les températures nocturnes ont été fixées à $7 \pm, 12 \pm$ et $17 \pm 1^{\circ} \mathrm{C}$. Le maintien de ces températures a été réalisé à l'aide de chaufferettes électriques de $4800 \mathrm{~W}$ contrôlées par des thermostats. Les chaufferettes étaient toutes munies de ventilateurs qui soufflaient l'air chaud dans des tubes de polyéthylène troué passant au milieu des plates-bandes. L'air de la serre au-dessus de l'écran thermique était maintenu juste au-dessus du point de congélation durant la nuit. Lors de journées claires et froides, toutes les parcelles atteignaient la température de nuit désirée en moins d'une heure après le coucher du soleil.

Les températures de 12 et $17^{\circ} \mathrm{C}$ ont pu être maintenues à toutes les nuits, depuis l'arrivée des plantes dans les unités expérimentales au 24 février jusqu'au 15 avril. Cependant, la température de $7^{\circ} \mathrm{C}$ n'a pu être maintenue lorsque les températures extérieures nocturnes dépassaient le point de congélation. Cette dernière situation ne s'est produite que quatre fois avant le 15 avril, les températures nocturnes des unités à $7^{\circ} \mathrm{C}$ se maintenant alors entre 8 et $10^{\circ} \mathrm{C}$. Au 15 avril, soit trois semaines avant le début de la récolte, les températures nocturnes de l'air ont été maintenues à $17 \pm 1^{\circ} \mathrm{C}$ dans toutes les unités expérimentales, ce qui signifie que toutes les plantes ont été soumises aux trois températures différentes du stade six feuilles et première grappe en bouton jusqu'au stade $75 \%$ des fleurs de la première grappe ouvertes dans le cas des plants au développement le plus retardé par les traitements, et jusqu'au stade première grappe complètement noué pour les plants les mieux développés. Durant toute la durée de l'expérience, la température diurne de l'air de toutes les platesbandes s'équilibre avec celle de la serre $\left(20 \pm 2^{\circ} \mathrm{C}\right)$ pendant le jour. La ventilation forcée est activée lorsque la température est supérieure à $25 \pm 2^{\circ} \mathrm{C}$ dans la serre.

Les récoltes ont été effectuées deux fois par semaine entre le 7 mai et le 20 août. Les fruits 
étaient classifiés en vendables et non-vendables, cette dernière catégorie comprenant les fruits difformes et les fruits verts. La date d'ouverture de la moitié des fleurs de la première grappe a aussi été notée pour chacune des trois tiges. Pour la troisième tige, cette date correspond à celle de la fin des traitements de température nocturne de l'air.

\section{RÉSULTATS ET DISCUSSION}

Les rendements en fruits totaux et vendables sont toujours significativement plus élevés pour les plants cultivés en NFT (tableau 1). Ce résultat était prévisible étant donné le meilleur contôle de l'environnement du système radiculaire avec la méthode NFT (RuddJones et Winsor 1978), ce qui s'avère particulièrement important dans le cas où, comme dans cette expérience, le temps de la production chevauche des périodes froides de l'année (Morgan et O'Haire 1978; Gosselin et Trudel 1983). D'autre part, les plants cultivés en sol ont fourni une plus faible proportion de fruits difformes ou immatures (tableau 1), nos résultats indiquant cependant que ces fruits non-vendables sont surtout constitués de fruits verts cueillis à la dernière date de récolte, les fruits difformes étant rares.

Contrairement aux résultats obtenus par plusieurs auteurs (e.g. Abdelhafeez et al. 1971; Moorby et Graves 1980; Orchard 1980; Papadopoulos et Tiessen 1983), les rendements en fruits totaux et vendables ont augmenté significativement avec la réduction des températures nocturnes de l'air (tableau 1). Ce résultat se vérifie aussi bien pour les plants cultivés en NFT que pour ceux cultivés en sol, quoique, dans ce dernier cas, ces mêmes rendements sont comparables lorsque la température de nuit est conservée à $17^{\circ} \mathrm{C}$ ou à $12^{\circ} \mathrm{C}$. Cependant, la compilation des rendements cumulatifs vendables par date de récolte pour chacune des six combinaisons de traitement sous étude montre que le refroidissement de la température nocturne de l'air retarde le début de la période de récolte (figure 1). Ce résultat est particulièrement apparent pour la culture en NFT, alors que la culture en sol montre des différences de précocité beaucoup moins marquées.
Si le rendement hâtif est défini comme le poids des fruits obtenu durant les trois premières semaines suivant le début de la récolte dans la première unité expérimentale (Yelle et al. 1987), alors l'avantage de chauffer la nuit afin d'obtenir les fruits le plus tôt possible devient évident, particulièrement pour le NFT (tableau 1). Trois semaines après le début de la récolte dans la plate-bande NFT $-17^{\circ} \mathrm{C}$, les plants conservés à $7^{\circ} \mathrm{C}$ de température de nuit n'avaient à peu près rien produit et ce, autant en sol qu'en NFT. Les plants ayant crû sous les températures nocturnes de l'air les plus élevées ont donc passé plus rapidement au travers de l'ensemble des étapes de développement menant aux fruits mûrs.

La date d'ouverture de $50 \%$ des fleurs de la première grappe de chacune des trois tiges est révélatrice à cet égard, car elle indique que le retard phénologique des plants gardés sous basses températures nocturnes de l'air atteint cinq jours en sol et 15 jours en NFT en ce qui a trait à la floraison (tableau 2). Ce retard passe à trois semaines à la fin de la période de grossissement des fruits formant le rendement hâtif (tableau 1), puis demeure relativement stable pour le reste de la saison de croissance (figure 1), les conditions de croissance de tous les plants ne différant dès lors que par le système de culture.

En effet, les traitements de température nocturne n'ont pu être maintenus que durant les deux premiers mois sur les sept qu'a duré cette production, soit durant la période de développement coüncidant avec la croissance en longueur des plantes et la phase d'ouverture des fleurs des premières grappes. Dès le milieu avril, il devenait de plus en plus difficile de maintenir la température nocturne de l'air à $7^{\circ} \mathrm{C}$, celle-ci étant dès lors maintenue constante à $17^{\circ} \mathrm{C}$ dans toutes les unités expérimentales. Le retard important en début de production, tel qu'exprimé par la date d'ouverture de $50 \%$ des fleurs de la première grappe (tableau 2) et par les rendements hâtifs (tableau 1), est donc causé par le facteur température nocturne de l'air, alors que le rattrapage des plants retardés par ces conditions 
Tableau 1. Rendements en fruits totaux, vendables et hâtifst, et proportion de fruits difformes ou immatures pour chacune des trois températures nocturnes de l'air et pour chacun des deux types de culture

\begin{tabular}{|c|c|c|c|c|c|}
\hline \multicolumn{2}{|c|}{ Traitement } & \multicolumn{3}{|c|}{ Rendements (kg plant ${ }^{-1}$ ) } & \multirow{2}{*}{$\begin{array}{c}\text { Pourcentage } \\
\text { de } \\
\text { fruits non-vendables } \\
\end{array}$} \\
\hline $\begin{array}{l}\text { Type de } \\
\text { culture }\end{array}$ & $\begin{array}{l}\text { Température } \\
\text { de nuit }\left({ }^{\circ} \mathrm{C}\right)\end{array}$ & Totaux & Vendables & Hâtifs & \\
\hline $\mathrm{NFT}_{\dagger}^{+}$ & $\begin{array}{r}7 \\
12 \\
17\end{array}$ & $\begin{array}{l}8.2 a \\
6.6 b \\
5.8 b\end{array}$ & $\begin{array}{l}7.3 a \\
6.5 a \\
5.3 b\end{array}$ & $\begin{array}{l}0.02 a \\
0.40 b \\
1.26 c\end{array}$ & $\begin{array}{r}11.0 a \\
1.5 c \\
7.8 b\end{array}$ \\
\hline Sol & $\begin{array}{r}7 \\
12 \\
17\end{array}$ & $\begin{array}{l}5.4 a \\
4.2 b \\
4.3 b\end{array}$ & $\begin{array}{l}5.0 a \\
4.0 b \\
4.2 b\end{array}$ & $\begin{array}{l}0.06 a \\
0.05 a \\
0.57 b\end{array}$ & $\begin{array}{l}6.0 a \\
4.0 b \\
2.5 b\end{array}$ \\
\hline $\begin{array}{l}\text { Moyennes } \\
\text { NFT } \\
\text { Sol }\end{array}$ & de culture $\ddagger$ & $\begin{array}{l}6.9 a \\
4.6 b\end{array}$ & $\begin{array}{l}6.4 a \\
4.4 b\end{array}$ & $\begin{array}{l}0.50 a \\
0.22 a\end{array}$ & $\begin{array}{l}7.0 a \\
4.0 b\end{array}$ \\
\hline Moyennes & $\begin{array}{c}\text { érature de nui } \\
7 \\
12 \\
17\end{array}$ & $\begin{array}{l}6.8 a \\
5.4 b \\
5.0 b\end{array}$ & $\begin{array}{l}6.2 a \\
5.2 a b \\
4.7 b\end{array}$ & $\begin{array}{l}0.04 a \\
0.18 a \\
0.86 b\end{array}$ & $\begin{array}{l}8.0 a \\
3.0 b \\
5.0 b\end{array}$ \\
\hline $\begin{array}{r}\text { Test de } F \\
\text { Type d } \\
\text { Tempér } \\
\text { Interact }\end{array}$ & $\begin{array}{l}\text { C) } \\
\text { uit (TN) } \\
\text { TN) }\end{array}$ & $\begin{array}{l}* * \\
* * \\
*\end{array}$ & $\begin{array}{l}* * \\
* * \\
* *\end{array}$ & $\begin{array}{l}* * \\
* * \\
* *\end{array}$ & $\begin{array}{l}* * \\
* * \\
* *\end{array}$ \\
\hline
\end{tabular}

†Rendement total obtenu durant les trois premières semaines suivant le début de la récolte. \$Comparaison de moyennes à l'intérieur des colonnes par le test de PLSD de Fisher. $5 \%$.

*, **Significatif au niveau 5 et $1 \%$, respectivement.

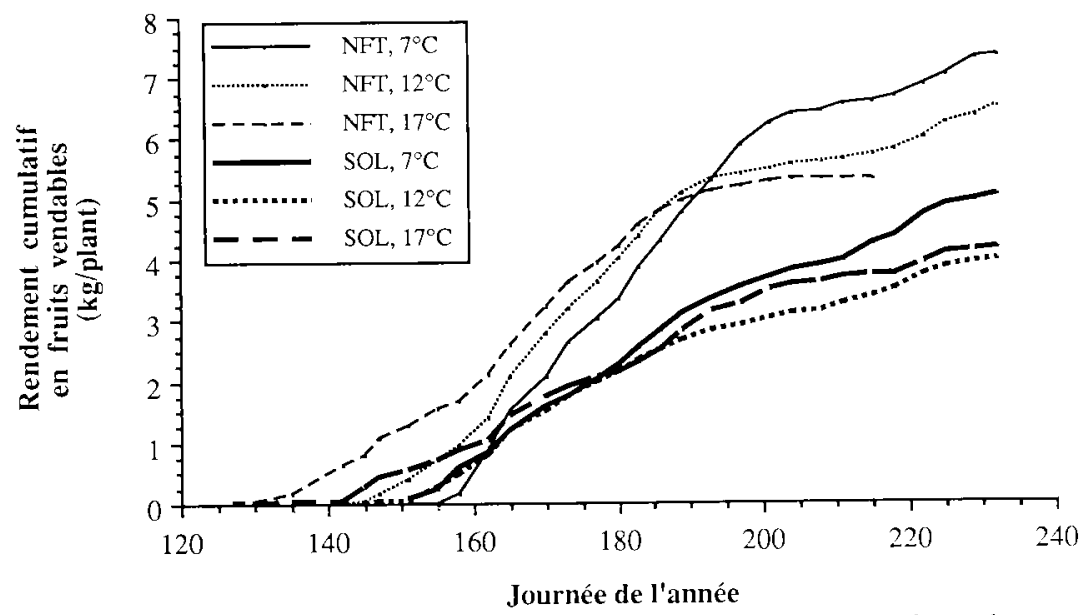

Figure 1. Influence des températures nocturnes de l'air et du type de culture sur le rendement cumulatif en fruits vendables d'une production de tomates de serre cultivées sur trois tiges sous petits tunnels.

de basse température, tel qu'exprimé par les rendements totaux et vendables (tableau 1 et figure 1), provient de cet ajustement de tous les plants traités aux conditions climatiques extérieures. Ces importants effets de la température nocturne de l'air sur la floraison et les rendements hâtifs, puis l'effet subséquent de rattrapage par les plants retardés en ce qui a trait aux rendements finaux, appuient l'hypothèse voulant que ce facteur contrôle la vitesse de l'ensemble des étapes liées au développement des plants de tomate (Morgan 
Tableau 2. Date d'ouverture de $50 \%$ des fleurs de la première grappe issue de chacune des tiges des plants cultivés sur trois tiges

\begin{tabular}{|c|c|c|c|c|c|c|}
\hline & \multicolumn{3}{|c|}{ NFT } & \multicolumn{3}{|c|}{ Sol } \\
\hline & $7^{\circ} \mathrm{C}$ & $12^{\circ} \mathrm{C}$ & $17^{\circ} \mathrm{C}$ & $7^{\circ} \mathrm{C}$ & $12^{\circ} \mathrm{C}$ & $17^{\circ} \mathrm{C}$ \\
\hline Tige 1 & $03-04$ & $29-03$ & $23-03$ & $02-04$ & $02-04$ & $29-03$ \\
\hline Tige 2 & $18-04$ & $09-04$ & $03-04$ & $17-04$ & $15-04$ & $12-04$ \\
\hline Tige 3 & $20-04$ & $12-04$ & $10-04$ & $26-04 \uparrow$ & $27-04 \uparrow$ & 一市 \\
\hline
\end{tabular}

†La première grappe de la troisième tige a avorté sous les conditions de culture en sol. Le résultat est alors pris sur la seconde grappe.

$\dagger \mathrm{La}$ troisième tige ne s’est pas allongée suffisamment pour permettre la formation d'une seule grappe de fleurs.

Tableau 3. Nombre de jours suivant la première journée de récolte (journée de l'année) pour que le rendement en fruits vendables atteigne $50 \%$ du rendement vendable observé à la fin de la saison de croissance

\begin{tabular}{|c|c|c|c|c|c|}
\hline & NFT & & \multicolumn{3}{|c|}{ Sol } \\
\hline $7^{\circ} \mathrm{C}$ & $12^{\circ} \mathrm{C}$ & $17^{\circ} \mathrm{C}$ & $7^{\circ} \mathrm{C}$ & $12^{\circ} \mathrm{C}$ & $17^{\circ} \mathrm{C}$ \\
\hline $\begin{array}{c}55 \\
(183)\end{array}$ & $\begin{array}{c}47 \\
(175)\end{array}$ & $\begin{array}{c}38 \\
(166)\end{array}$ & $\begin{array}{c}56 \\
(184)\end{array}$ & $\begin{array}{c}49 \\
(177)\end{array}$ & $\begin{array}{c}51 \\
(179)\end{array}$ \\
\hline
\end{tabular}

et O'Haire 1978; Papadopoulos et Tiessen 1983).

La mesure du nombre de jours pour que les plantes fournissent la moitié de leur production totale, laquelle indique en fait la rapidité des plants à se libérer de leurs fruits, montre toutefois que le retard pris par les plants conservés sous de basses températures nocturnes de l'air se manifeste durant une grande partie de la saison de croissance, particulièrement sous les conditions de la culture en NFT (tableau 3). En effet, les plants croissant en NFT sous des températures de nuit de $17^{\circ} \mathrm{C}$ n'ont eu besoin que de 38 jours pour fournir la moitié de leur production totale, alors que ceux conservés à $7^{\circ} \mathrm{C}$ ont exigé 17 jours de plus. Cet effet est beaucoup moins important pour la culture en plein sol, la différence entre les extrêmes variant de 49 jours à la température de nuit de $12^{\circ} \mathrm{C}$ à 56 jours à $7^{\circ} \mathrm{C}$. Un contrôle moins précis des conditions de l'environnement du système radiculaire explique fort probablement ce dernier résultat. Ces mêmes données indiquent aussi que le temps requis pour fournir la moitié de la récolte est le même pour la culture en NFT et pour la culture en sol lorsque les températures de nuit sont conservées à 7 ou à $12^{\circ} \mathrm{C}$. L'augmentation des rendements introduits par l'utilisation de la méthode NFT se transmet donc uniformément tout au long de la saison de croissance, la vitesse de développement des plants étant aussi rapide dans un système de culture que dans l'autre. Quant aux résultats à $17^{\circ} \mathrm{C}$ de température de nuit, il s'explique par le fait que la troisième tige n'a jamais voulu s'allonger dans ce cas-ci, alors qu'il est reconnu que les deux premières grappes fournissent toujours la plus grande partie des fruits récoltés sur la troisième tige (Gallagher et al. 1981).

En somme, les plants cultivés sur trois tiges sous petits tunnels et conservés aux températures de nuit les plus chaudes ont donc fourni la majorité de leurs fruits alors que les conditions climatiques extérieures se rapprochaient le plus du printemps hâtif, alors que ceux conservés aux températures les plus froides les ont fourni lorsque les conditions climatiques extérieures étaient trop clémentes pour permettre le maintien de ces basses températures de nuit. De plus, ces derniers n'ont pas fourni leurs fruits dans un laps de temps aussi court que ceux à température de nuit plus élevée, ce qui a permis à la production de se prolonger dans le temps et d'ainsi fournir un meilleur rendement final en fruits totaux et vendables. Il est important de 
constater à cet égard que le rendement des plants croissant à des températures nocturnes plus basses n'a rejoint celui des plants croissant la nuit à $17^{\circ} \mathrm{C}$ qu'au moment où la récolte cessait sur ceux-ci (figure 1).

Ces facteurs de précocité et de rapidité à fournir les fruits sont importants, car l'objectif du producteur lors d'une production dite hâtive n'est pas seulement d'obtenir les rendements les plus élevés possibles en fruits venables, mais aussi de produire le plus tôt possible, lorsque les prix de vente et la demande sont les plus élevés. En contrepartie, le chauffage des serres dans les régions froides représente, avec la main-d'oeuvre. la plus grande part des charges variables de production, de sorte qu'il peut tout de même être préférable d'accepter une récolte plus tardive avec un rendement plus élevé.

La culture sur trois tiges en NFT sous petits tunnels semble donc bien adaptée à la problématique de production de petits complexes de serres situés dans des communautés nordiques isolées. L'intérêt de réduire les températures nocturnes de l'air pour des fins d'economie d'énergie n'est cependant pas évident. Les rendements en fruits totaux et vendables des plants qui se sont développées pendant deux mois sous de basses températures sont certes plus élevés, sauf qu'ils s'expliquent par la longue période de culture et les meilleures conditions de croissance et de développement durant la période de grossissement de la majorité des fruits. De tels traitements réduisent passablement la vitesse de développement des plants et, conséquemment, les rendemets hâtifs, alors qu'ils augmentent le temps requis pour qu'un plant fournisse la moitié de ses fruits. De plus, l'application des traitements de basses températures de nuit pendant une période plus longue que deux mois aurait assurément amplifié ce retard. Il y aurait donc intérêt à établir un rapport économique tenant compte des gains en coûts de chauffage et en rendement engendrés par la réduction des températures de nuit, mais aussi des pertes en coûts de fonctionnement occasionnées par une production qui prend plus de temps à fournir le même rendement.

\section{REMERCIEMENTS}

Les auteurs remercient Agriculture Canada et son superviseur scientifique, M. Harold Jackson, pour l'aide financière apportée à la réalisation de cette étude (contrat 2OSU.01843-1-EC12) et MM. Jacques Allaire, Daniel Gagnon et George Gallagher pour leur assistance technique et scientifique.

Abdelhafeez, A. T., Harssema, H., Veri, G. et Verkerk, K. 1971. Effects of soil and air temperature on growth, development and water use of tomatoes. Neth. J. Agric. Sci. 19: 67-75.

Calvert, A. 1964. The effects of air temperature on growth of young tomato plants in natural light conditions. J. Hortic. Sci. 39: 194-211.

Cooper, A. 1979. The ABC of NFT. Grower Books, London, U.K.

Conseil de productions végétales du Québec. 1984. Légumes de serre: culture. Agdex 290/20. CPVQ, ministère de l'Agriculture, des Pêcheries et de l'Alimentation du Québec. $156 \mathrm{pp}$.

Gallagher, G. et Vonarburg, J. J. 1981. Heat energy comparison between a two layered conventional plastic greenhouse and a greenhouse utilising the heat shielded interior plastic tunnel concept. Rapport à Agriculture Canada, contrat 07SZ-01843-0-01915, $50 \mathrm{pp}$.

Gallagher, G., Vonarburg J. J. et St-Pierre, C. 1981. Étude comparative du comportement d'une culture de tomate dans une serre à double tunnel et dans une serre conventionnelle. Rapport à Agriculture Canada, contrat 07SZ-01843-0-01915, $35 \mathrm{pp}$.

Gosselin, A. et Trudel, M. J. 1983. Interactions between air and root temperatures on greenhouse tomato: 1. Growth, development and yield. J. Am. Soc. Hortic. Sci. 108: 901-905.

Hussey, G. 1965. Growth and development in the young tomato. 3 . The effect of night and day temperatures on vegetative growth. J. Exp. Bot. 16: 373-385.

Ingrata, F . 1980. Reducing night temperature by soil warming. Greenhouse Veg. Newsl., Ontario Ministry of Agriculture and Food, Toronto, Ont. 3-80.

Jones, D. A. G., Sandwell, I. et Talent, D. J. W. 1978. The effect of soil temperature when associated with low air temperature on the cropping of early tomatoes. Acta Hortic. 76: 167-171. Kaname, T. et Itagi, T. 1973. Effects of various air and soil temperatures on the growth and yield of tomato under glass and plastic house culture. Bul. Kanagawa Hortic. Exp. Sta. 21: 67-76. 
Lewis, D. 1953. Some factors affecting flower production in the tomato. J. Hortic. Sci. 28: 207-219.

Moorby, J. et Graves, C. J. 1980. Root and air temperature effects on growth and yield of tomatoes and lettuce. Acta hortic. 98: 29-43.

Morgan, J. V. et O'Haire, R. 1978. Heated hydroponic solutions as an energy saving technique. Acta Hortic. 76: 173-180.

Orchard, B. 1980. Effect of root and air temperature on growth and yield of tomatoes. Acta Hortic. 98: 19-28.

Papadopoulos, A. P. et Tiessen, H. 1983. Root and air temperature effects on the flowering and yield of tomato. J. Am. Soc. Hortic. Sci. 198: 805-809.

Rudd-Jones, D. et Winsor, G. W. 1978. Environmental control in the root zone: nutrient film culture. Acta Hortic. 87: 185-195.

Verkerk, K. 1966. Temperature response in early tomato production. Acta Hortic. 4: 26-31.

Yelle, S., Gosselin, A. et Trudel, M. J. 1987. Effets à long terme de l'enrichissement carboné sur la tomate de serre cultivée avec ou sans éclairage d'appoint. Can. J. Plant Sci. 67: 899-907. 